\title{
The rate of the musculoskeletal pathology assessment for military personnel and civil population
}

\author{
L. Plavina ${ }^{1}$, H. Karklina ${ }^{1}$, and I.Viksne ${ }^{2}$ \\ ${ }^{1}$ Rīga Stradiņš University, Riga, Latvia \\ ${ }^{2}$ General practice Doctorate of Riga region, Riga, Latvia
}

\begin{abstract}
Musculoskeletal disorders are an important cause of morbidity for civil population as well as for military personnel. Musculoskeletal disorders represent a prevalent source of patient visits, lost work time, hospitalization and disability. The three most important pain areas are those of lumbar spine, shoulders and lower extremities. We provide assessment of Anthropometric characteristics in two groups. Anthropometric characteristics of military personnel $(n=60)$ that leave the active service and civil population of the same age $(n=100)$ were collected during two year period (2011/2012). The anthropometric characteristics were evaluated for military personnel going for retirement with diagnosis of musculoskeletal pathology. The evaluated anthropometric characteristics were the height, the body mass and the anthropometric indices. Body fat ranges for standard adults were calculated according to NIH/WHO BMI guidelines. $30 \%$ of military persons with diagnosis of musculoskeletal pathology and individuals from civil population group with diagnosis of the musculoskeletal pathology have values of body mass index above the standard level One of the reasons the musculoskeletal pathology is the overweight it may be important to provide preventive measures to reduce the risk of musculoskeletal disorders linked to specific job and treatment at early stage.
\end{abstract}

\section{Introduction}

Musculoskeletal pathology is one of the most common work-related health problems [1-3], as well as an important cause of morbidity for both civil population and military personnel [5-7]. It is an inflammatory and degenerative ailment that affects all individuals at working age.

Musculoskeletal pathologies are linked to extrinsic factors such as inappropriate biomechanical loads, vibration, organizational relationships, psychosocial climate and culture, as well as work-related cognitive, sensory and psychosocial load [8, 9]. It is also related to intrinsic factors such as body built, body composition, anthropometric characteristics, and inheritance. Psychosocial factors may play an important role in development exacerbation and maintenance of work disability in both civil and military population [10-12].

Musculoskeletal disorders represent a prevalent source of patient visits, lost work time, hospitalization and disability. That composes expenses between 90 and 350 billion EURO in Latvia [13]. According to data of wide European occupational health survey, approximately $46 \%$ of European workers reported back pain, while $43 \%$ had pain in the shoulder, neck and upper limb muscles [14]. 
In military environment this pathology is frequent considering specific work conditions that comprise non-mechanical risk factors such as: environmental factors (exposure to cold, glare, reflections); work organisation (monotonous or repetitive work, no job control); equipment-related factors (accessibility, usability and comfort, adaptation to user anthropometry, physiology of motion and set movements); psychosocial factors (climate and culture, workplace relationships, support, job satisfaction).

Due to heavy physical load on daily basis, musculoskeletal pathology is more common in military personnel than in civil population.

Musculoskeletal pathology has social and economic consequences. Large number of workers is not able to fulfil certain tasks and is forced to stop working before retirement age. Chronic musculoskeletal disorders result in disabilities that require long-term rehabilitation and functional impairment leading to premature discharge from military service.

Nevertheless, it may be significant to provide preventive measures to reduce the risk of musculoskeletal disorders that would allow efficient treatment at early stage.

Following hypothesis of the research was proposed: diseases of the muscular and skeletal system are more frequent in military persons than in civilians.

\section{Aim}

The aim of the study is to provide analysis of anthropometric characteristics in accordance with the rate of diagnosis of musculoskeletal pathologies in two groups: civil population and military personnel.

\section{Material and methods}

The study was carried out in a five-year period (2008-2012) applying a randomized selection of civil and military personnel groups.

60 male respondents who had retired from active service in the National Armed Forces of Latvia were recorded and anthropometrically measured. Their active service had lasted between 7 and 20 years. Similarly, anthropometric measurements (length, weight, body mass index, anthropometrical indices) of civilians (male; $n=100$ ) were analysed. Furthermore, fat content was defined in accordance with WHO guidelines, and disease epidemiology of musculoskeletal system was investigated [4]. Anonymous survey also provided with data about smoking frequency rates in groups under the study.

The military personnel were divided into two groups: respondents with diagnosis of musculoskeletal pathology (in medical card) and respondents without the respective diagnosis. The study provides analysis of the anthropometric characteristics of body mass and body height, as well as calculation of the body mass index.

The diagnosis of musculoskeletal pathology was documented in individual medical card and was grouped according to Classification ICD-10 (M47.9, M54.5 and G54.5).

\section{Results and discussion}

Results showed that musculoskeletal pathology is a dominated pathology for military personnel during the last decade (Fig. 1).

$20 \%$ of respondents from military personnel without diagnosis of musculoskeletal pathology had the body mass index in a standard interval $\left(18.5-24.9 \mathrm{~kg} / \mathrm{m}^{2}\right), 55 \%$ were included into overweight group according to the body mass index value (from 24.9$29.9 \mathrm{~kg} / \mathrm{m}^{2}$ ), while for the rest of respondents (i.e. $25 \%$ ) adiposity problems (body mass index value were over $30.0 \mathrm{~kg} / \mathrm{m}^{2}$ ) were identified. 


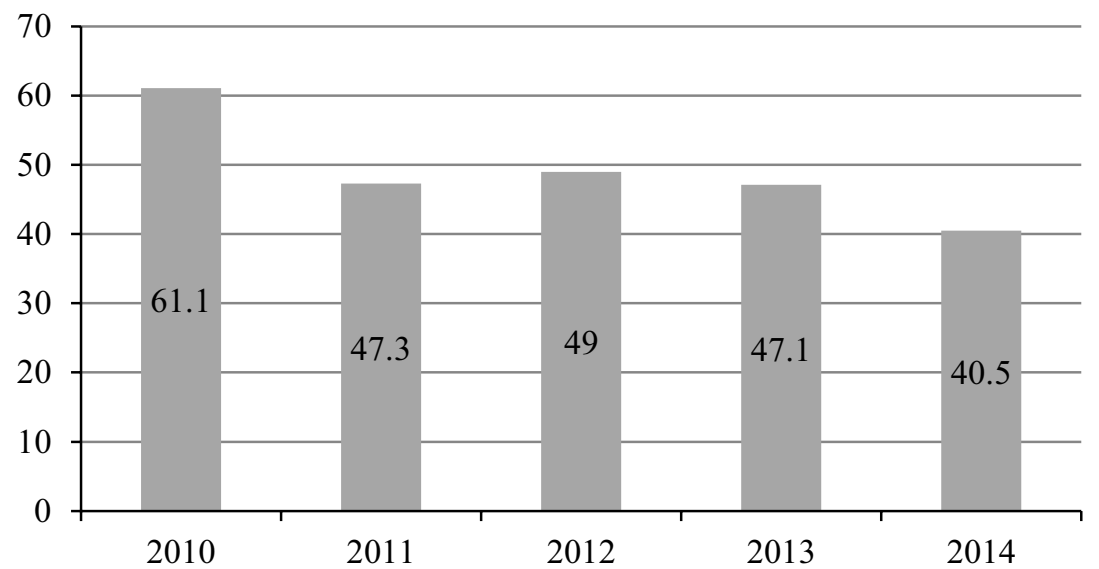

Figure 1. Diagnosis of musculoskeletal pathology for retired military persons (\%) according data of central medical expert commission.

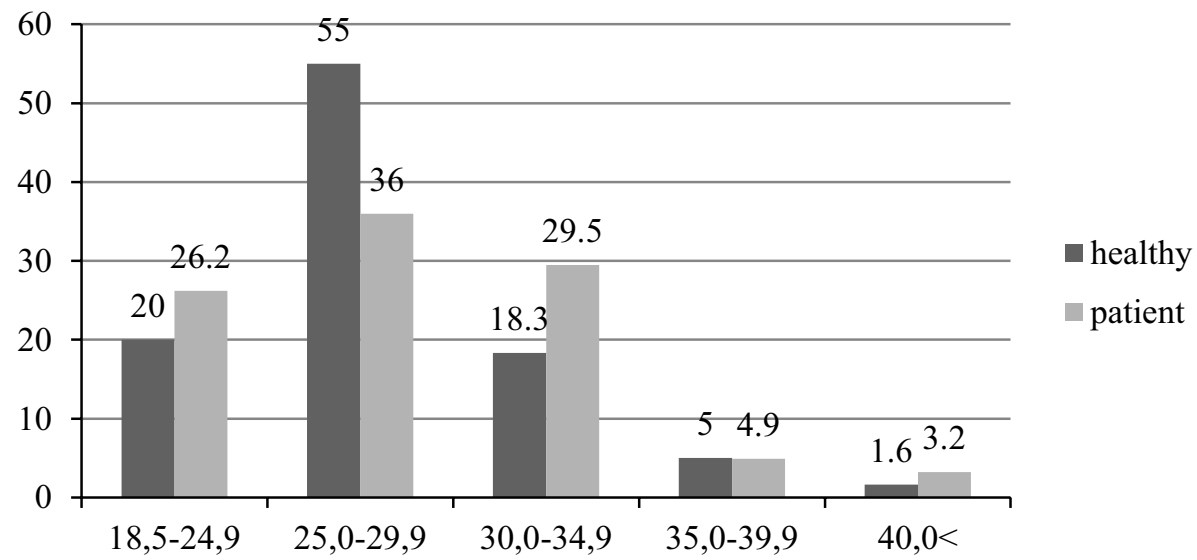

Figure 2. BMI characteristics for military persons with and without musculoskeletal pathology.

Body mass index for $26.2 \%$ of military personnel respondents with diagnosis of musculoskeletal pathology corresponds to a standard interval $\left(18.5-24.9 \mathrm{~kg} / \mathrm{m}^{2}\right), 36 \%$ of respondents were included into overweight group, while $37.6 \%$ of respondents showed a high adiposity index (BMI above $30.0 \mathrm{~kg} / \mathrm{m}^{2}$ ) (Fig. 2).

The age is one of the leading factors, which increases the value of body mass index. Respondents were distributed according to the age (Fig. 3). It was observed that respondents with musculoskeletal pathologies have higher value of the body mass index, however with age the difference slightly slip down. In the age group of 30 to 40 years the body mass index for individuals with musculoskeletal pathologies is higher, however in the age group after 50 years the difference is minimal.

Analysis of the anthropometric characteristics in the civil population group shows that approximately $31.4 \%$ of civil individuals without diagnosis of musculoskeletal pathology has the body mass index in a standard interval $\left(18.5-24.9 \mathrm{~kg} / \mathrm{m}^{2}\right)$. Also there are no individuals with standard value of the body mass index who are diagnosed with musculoskeletal pathology. Value of the body mass index in the civil group with diagnosis of musculoskeletal 


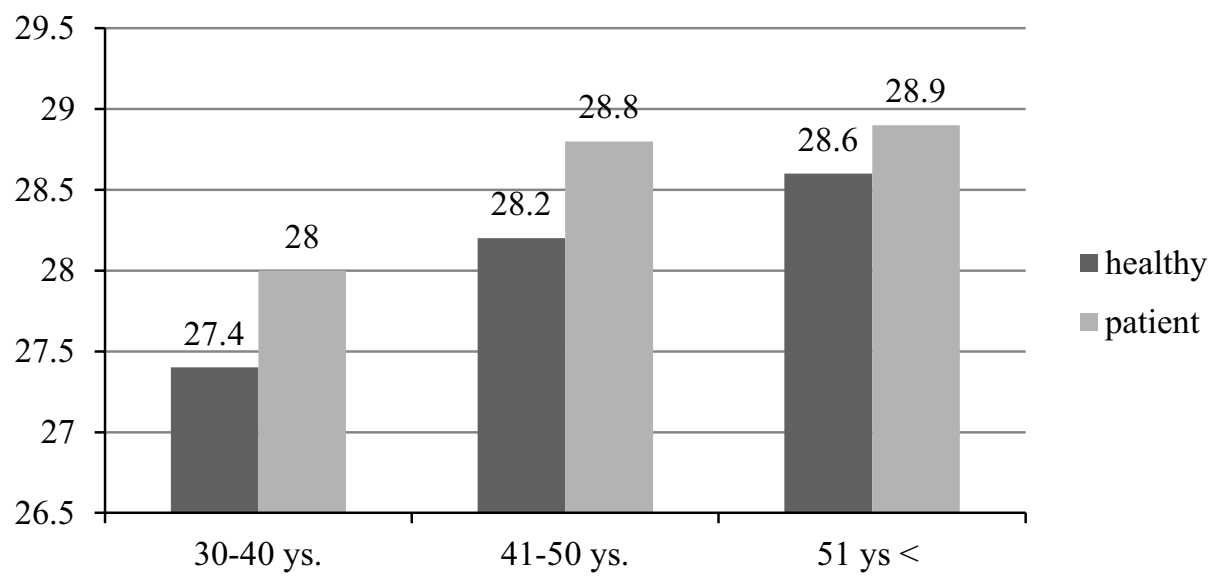

Figure 3. Distribution of BMI characteristics in various age groups of military persons with and without musculoskeletal pathology.

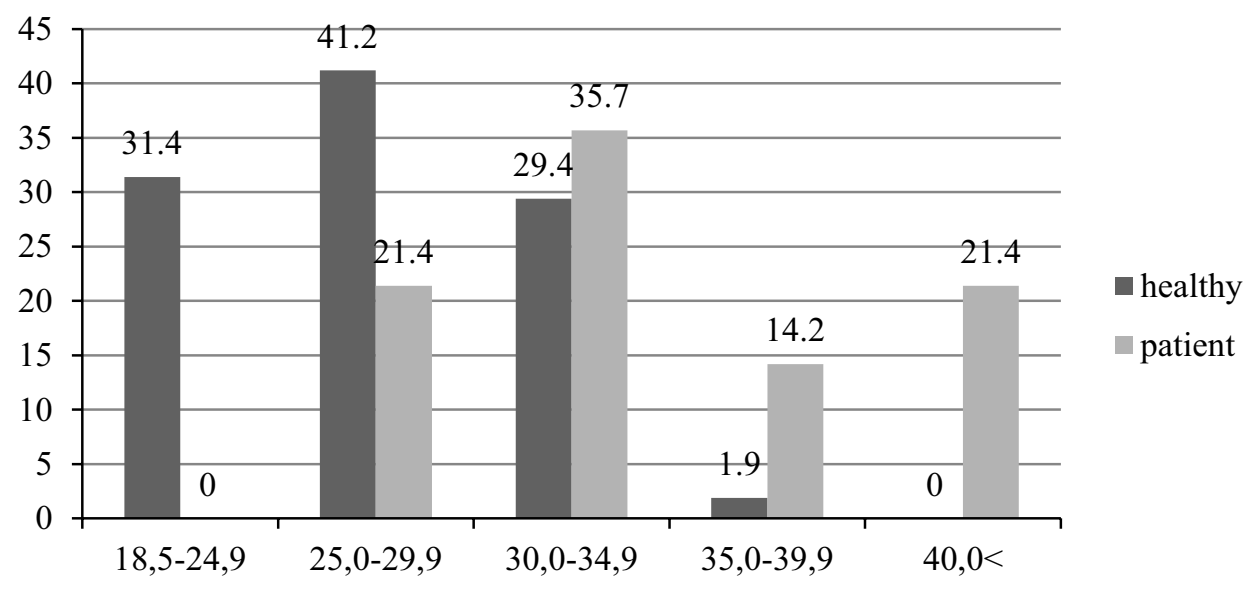

Figure 4. BMI characteristics for civil persons with and without musculoskeletal pathology.

pathology indicates that approximately $71.3 \%$ of respondents have adiposity problems. Adiposity problems for civil population respondents with no musculoskeletal pathology are two times less frequent.

Analysis of the value of body mass index and aging was produced. Distribution of values of the body mass index shows a slight tendency of increased body mass value in the group of civil individuals with musculoskeletal pathology diagnosis (Fig. 5). The value of body mass index in the age group from 30 to 40 years is about $20 \%$ higher for individuals with musculoskeletal pathology than in the group of civil individuals with no musculoskeletal pathology.

The difference between the values of body mass index equalize in the age group from 51 to 60 years. Adiposity problems were recognized in all age groups under discussion for respondents with diagnosis of the musculoskeletal pathology.

The analysis on distribution of the value of body mass index in case of both civil respondents and military respondents groups without musculoskeletal pathology was carried out. 


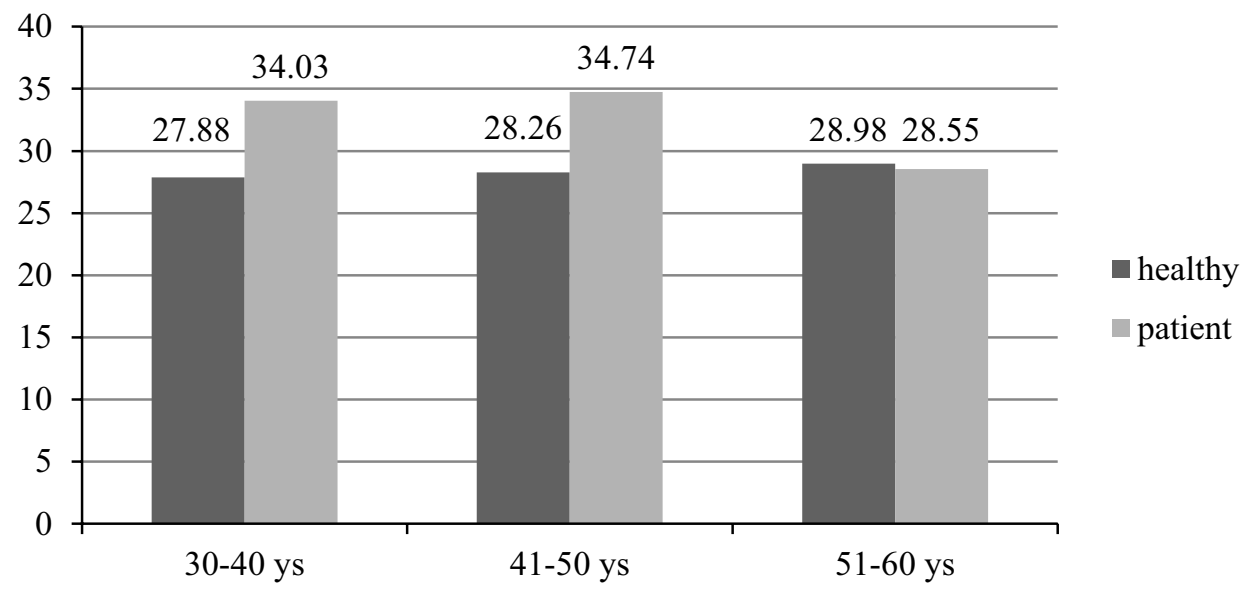

Figure 5. Distribution of BMI characteristics in various age groups for civil persons without and with musculoskeletal pathology.

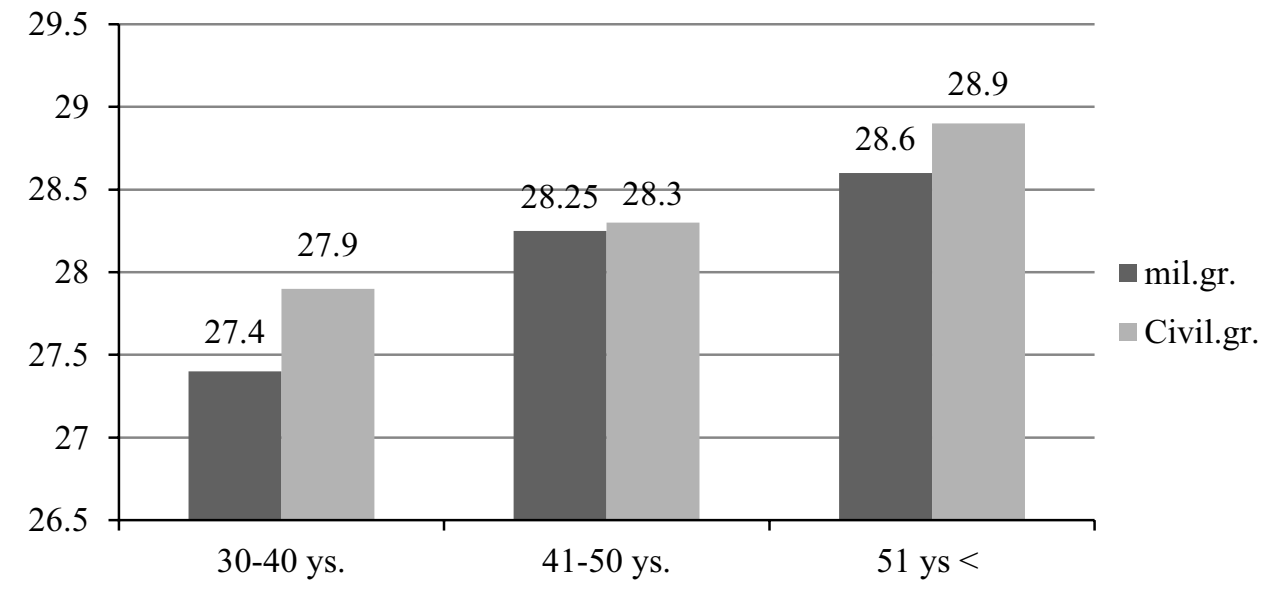

Figure 6. Distribution of BMI characteristics in various age groups for individuals without musculoskeletal pathology in both military and civil groups.

Specific military conditions have an impact on the value of body mass index in all age groups of civil population. The difference in the value of body mass index is higher in the group of 30 to 40 years and they slightly decrease in the group of 51 to 60 years for respondents without musculoskeletal pathologies (Fig. 6), as well as in the group of respondents with musculoskeletal pathologies (Fig. 7).

Distribution of the value of body mass index in various age groups for both military and civil respondents with diagnosis of the musculoskeletal pathology shows that increased BMI in civil persons group is for about $20 \%$ higher. The values of body mass index were higher in all civil-person groups than in the military-person group.

The hypothesis posed at the beginning of the study was partially proven as correct, i.e. the number of men with musculoskeletal pathology in the age groups under the study is almost equal regardless of their occupation. 


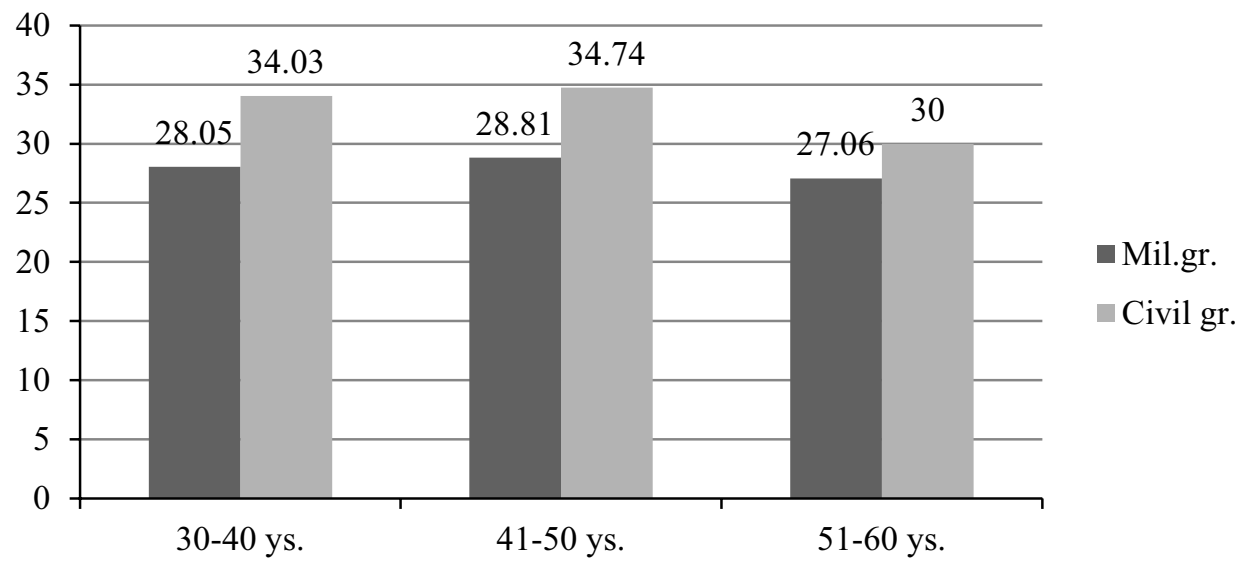

Figure 7. Distribution of BMI characteristics in various age groups for military and civil persons with musculoskeletal pathology.

\section{Conclusion}

1. The values of body mass index correspond to the standard level only in $20 \%$ of cases in the group of military respondents without musculoskeletal pathology. 55\% of military persons without musculoskeletal pathology have overweight problems, while adiposity problems were identified in $25 \%$ of cases.

2. $36 \%$ of military persons with diagnosis of the musculoskeletal pathologies have overweight problems; $37.5 \%$ were included into the adiposity group.

3. Musculoskeletal pathology is provoked by external (environmental) and inner (individual) risk factors. Overweight in combination with other external factors, which are related to specific work conditions, could be one of the risk factors in developing musculoskeletal pathology.

4. $30 \%$ of military persons with diagnosis of musculoskeletal pathology and individuals from civil population group with diagnosis of the musculoskeletal pathology have values of body mass index above the standard level.

5. The value of body mass index for both civic individuals with and without diagnosis of musculoskeletal pathologies was higher than in the group of military personnel.

\section{References}

[1] S.M. Berkowitz, M. Feuerstein, M.S. Lopez, C.A. Peck, Jr Military Medicine, 164, 6: 412-8 (1999).

[2] D. Glad, E. Skillgate, L.W. Holm, Eur Spine J., Apr; 21, 4: 739-44 (2012).

[3] M. Feuerstein, S. Berkowitz, C.A. Peck Jr, Med J. of Occupational \& Environmental Medicine, 39, 1: 68-78 (1997).

[4] D. Gallagher, S.B. Heymsfield, M. Heo, S.A. Jebb, P.R. Murgatroyd, Y. Sakamoto, Am J ClinNutr, 72, 3: 694-701 (2000).

[5] T. Heir, G. Eide, Scand. Med Sci Sports, 7, 5: 304-11 (1997).

[6] T. Heir, G. Eide, Scand. J, Med Sci Sports, 6, 4: 222-7 (1996).

[7] I. Kuorinka, B. Jonsson, A. Kilbom, H. Vinterberg, F. Biering-Sфrensen, G. Andersson, K. Jфrgensen, Applied Ergonomics, 18, 3: 233-237 (1987).

[8] P.A. Leggat, D.R. Smith, Jr of Musculoskeletal pain, 15, 2: 25-32 (2007). 
[9] J. Parkkari, H. Taanila, J. Suni, V.M. Mattila, O. Ohrankämmen, P. Vuorinen, P. Kannus, H. Pihlajamaki, BMC Medicine, 9: 35. (http://www . biomedcentral. com/1741-7051/8/35) (2011).

[10] L. Rosendal, H. Langberg, A. Skov-Jensen, M. Kjaer, Clin J Sport Med., 13: 157-163 (2003).

[11] H. Taanila, J. Suni, H. Pihlajamäki, V.M. Mattila, O. Ohrankämmen, P. Vuorinen, J. Parkkari, BMC Musculoskelet Disorders, 11: 146. (http://www . biomecentrral. com/1741-7-51/9/35) (2010).

[12] H. Taanila, J. Suni, H. Pihlajamäki, V.M. Mattila, O. Ohrankämmen, P. Vuorinen, J. Parkkari, BMC Musculoskeletal Disorders, 10: 89 (http: //www . biomecentrral . com/1741-24474/10/89) (2009).

[13] K. Zheltoukhova, R. Thomas, S. Bevan, Fit For Work (2012) (latv.).

[14] R. Gauthy, Musculoskeletal system disorders/an ill-understood "pandemic" (2007). 